\title{
Studying signal collection in the punch-through protection area of a silicon micro-strip sensor using a micro-focused X-ray beam
}

\author{
L. Poley ${ }^{\mathrm{a}}$, R. Bates ${ }^{\mathrm{b}}$, I. Bloch ${ }^{\mathrm{a}}$, A.J. Blue ${ }^{\mathrm{b}}$, V. Fadeyev ${ }^{\mathrm{c}}$, L. Meng ${ }^{\mathrm{d}}$, L. Rehnisch ${ }^{\mathrm{e}}$, M. Stegler ${ }^{\mathrm{a}}$, Y. \\ Unno $^{\mathrm{f}}$ \\ ${ }^{a}$ Deutsches Elektronen-Synchrotron (DESY), D-15738 Zeuthen, Germany \\ ${ }^{b}$ SUPA School of Physics and Astronomy, University of Glasgow, Glasgow G12 8QQ, United Kingdom \\ ${ }^{c}$ Santa Cruz Institute for Particle Physics (SCIPP), University of California Santa Cruz, Santa Cruz, CA 95064, \\ USA \\ ${ }^{d}$ Oliver Lodge Laboratory, University of Liverpool, Liverpool L69 3BX, United Kingdom \\ ${ }^{e}$ Institut für Physik, Humboldt-Universität zu Berlin, D-12489 Berlin, Germany \\ ${ }^{f}$ Institute of Particle and Nuclear Study, High Energy Accelerator Research Organization (KEK), 1-1 Oho, \\ Tsukuba-shi, Ibaraki-ken 305-0801, Japan
}

\begin{abstract}
For the Phase-II Upgrade of the ATLAS detector [1], a new, all-silicon tracker will be constructed in order to cope with the increased track density and radiation level of the High-Luminosity Large Hadron Collider. While silicon strip sensors are designed to minimise the fraction of dead material and maximise the active area of a sensor, concessions must be made to the requirements of operating a sensor in a particle physics detector. Sensor geometry features like the punch-through protection deviate from the standard sensor architecture and thereby affect the charge collection in that area. In order to study the signal collection of $\mathrm{n}^{+}-\mathrm{p}^{-}-\mathrm{p}^{+}$silicon strip sensors over their punch-throughprotection area, ATLAS silicon strip sensors were scanned with a micro-focused X-ray beam at the Diamond Light Source. Due to the highly focused X-ray beam $\left(2 \times 3 \mu \mathrm{m}^{2}\right)$ and the short average path length of an electron after interaction with an X-ray photon $(\leq 2 \mu \mathrm{m})$, local signal collection in different sensor areas can be studied with high resolution. This study presents results of high resolution 2D-scans of the punch-through protection region of ATLAS silicon micro-strip sensors, showing how far the strip signal collection area extends toward the bias ring and how the region is affected by radiation damage.
\end{abstract}

Keywords: ATLAS, silicon strip sensors, radiation damage, punch-through protection

\section{Introduction}

Silicon sensors for the ATLAS strip tracker 10 are designed to be operated in the high- ${ }_{11}$ radiation environment of the High-Luminosity 12 Large Hadron Collider 2 .

A structured gap between strip implant ${ }_{14}$ and bias ring (punch-through protection) pro- ${ }_{15}$

Email address: Anne-Luise.Poley@Desy.de ()

tects readout electronics from high currents induced by beam splashes [3. While this gap is short compared to the length of a sensor strip $(16.2 \mu \mathrm{m}$ compared to about $2.4 \mathrm{~cm}$ strip length, i.e. less than $0.1 \%$, for modules with short sensor strips), it will make up a combined area of about $800 \mathrm{~cm}^{2}$ on the 18,000 detector modules foreseen to be built for the ATLAS strip tracker [4], corresponding to the area of eight modules. 


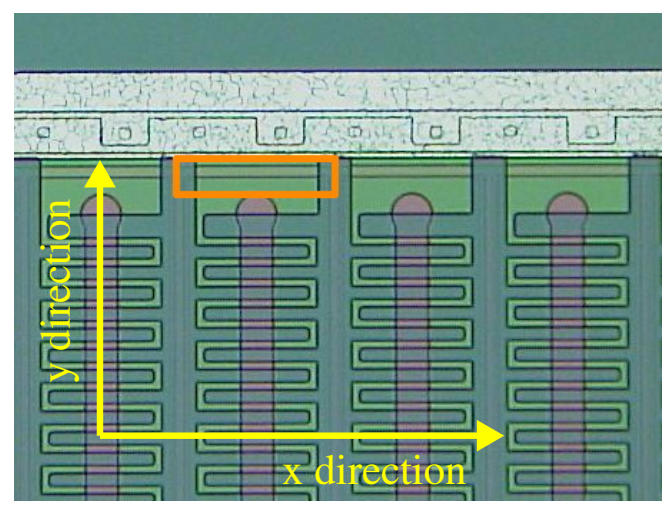

Figure 1: Structured gap between strip implants 55 (brown) and bias ring (grey) with unknown signal col- 56 lection (marked orange for individual sensor strip).
For efficient tracking, the strip tracker is designed to minimise gaps between the active sensor areas of adjacent modules. Inefficient charge collection in the punch-through protection region would effectively increase the amount of inactive material surrounding the active sensor area and hence reduce the detector performance.

It was thus important to study how far a sensor strip can collect signals beyond the end of its implant (see figure 1).

\section{Experimental setup}

Studying this sensor region requires a position resolution $\mathcal{O}(\mu \mathrm{m})$, which was achieved by using a micro-focused X-ray beam $\left(2 \times 3 \mu \mathrm{m}^{2}\right)$ and $\mu \mathrm{m}$ precision positioning stages at the Diamond Light Source beamline B16 [5].

Given the dimensions of the implant width 76 $(16 \mu \mathrm{m})$ and the strip pitch $(75.5 \mu \mathrm{m})$, the 77 micro-focused beam was well suited to study 78 the strip responses in different areas.

The Diamond Light Source provides photons 80 every 2 ns. Using only monochromatic $15 \mathrm{keV} \quad 81$ photons reduces the flux to $1.2 \pm 0.2$ photons 82 arriving within $10 \mathrm{~ns}$. A $15 \mathrm{keV}$ photon has a 83 $51 \%$ probability to react within $300 \mu \mathrm{m}$ of sili- 84 con, so that each recorded event (sampling time 85 $25 \mathrm{~ns}$ ) contains deposited charge from $1.4 \pm 0.386$

\section{Performed measurements}

The main challenge of this study was locating the position of the gap between implant and bias ring prior to the measurement. In order to obtain sufficient statistics at each sensor position, a data acquisition time of about $2.5 \mathrm{~min}$ was required (corresponding to 50,000 events). With the chosen step sizes (see table1), the allocated beam time allowed to scan a length of $170 \mu \mathrm{m}$ along the sensor strip. In order to scan across a gap with a length of $16 \mu \mathrm{m}$, the scan thus required a positioning precision of about 


\begin{tabular}{l|cc} 
& Coarse scan & Fine scan \\
\hline Step size $x,[\mu \mathrm{m}]$ & 15 & 15 \\
Scan length $\mathrm{x},[\mu \mathrm{m}]$ & 240 & 240 \\
Step size $\mathrm{y},[\mu \mathrm{m}]$ & 50 & 5 \\
Scan length $\mathrm{y},[\mathrm{mm}]$ & $1.6 / 1.7$ & $0.085 / 0.12$ \\
Number of events & 50,000 & 50,000
\end{tabular}

Table 1: Parameters for coarse and fine scans of unirradiated/irradiated sensor

$\pm 80 \mu \mathrm{m}$ for this scan. The sensor was positioned with respect to the beam using a positioning laser with a positioning uncertainty of about $\pm 500 \mu \mathrm{m}$, which was insufficient for the required precision.

A method was conceived for the precise location of the end of the strip implant using sensor features: in previous studies, aluminium bond pads on sensors (AC pads) had been found to affect the response of sensor strips [8]. In the vicinity of bond pads on a sensor strip, this strip collects charges over a larger area than sensor strips without bond pads. Bond pads are large $\left(200 \times 56 \mu \mathrm{m}^{2}\right)$ compared to the punch-through protection region and thus can be found and mapped in a coarse scan over a large sensor area. Since the position of the punch-through protection region with respect to the position of $\mathrm{AC}$ pads is known from the sensor geometry, the location of AC pads allows the subsequent location of the end of the strip implant.

Scans were thus performed in two stages (see table 1): in a first step, a coarse scan was performed over a comparably large sensor area. The data analysis of this scan was used to locate the positions of $\mathrm{AC}$ pads on the sensor and extrapolate the positions of bias ring and structured gap. Afterwards, a second scan was set up using the coordinates determined in the first step.

Figures 2a and 2b show the hit maps obtained in the coarse sensor scans. Using the

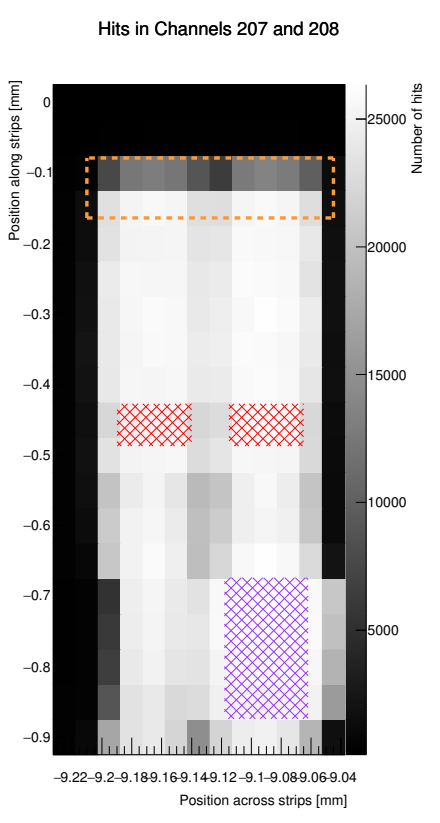

(a) Unirradiated sensor Hits in Channels 97 and 98

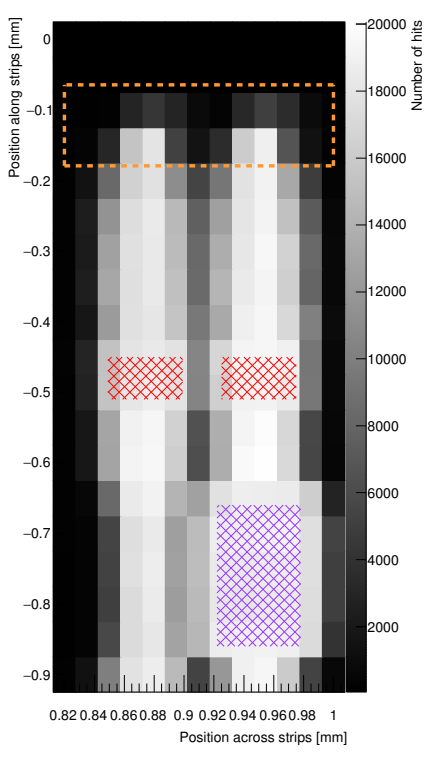

(b) Irradiated sensor

Figure 2: Hit maps from coarse scans on an unirradiated sensor (left) and an irradiated sensor (right), showing hits from two adjacent channels each. The hit patterns allow to identify AC pads (violet) and DC pads (red) and to extrapolate the position of the gap between implant and bias ring (marked yellow). 
reconstructed positions of $\mathrm{AC}$ pads from the coarse scan, the positions of bias ring and end of strip implant were determined with a precision of $\pm 20 \mu \mathrm{m}$.

\section{Results}

Hit maps obtained at the end of strip implants showed few hits being collected between the end of the strip implant and the bias ring, both for an unirradiated sensor (see figure 3a) and an irradiated sensor (see figure 3b). Figures $4 \mathrm{a}$ and $4 \mathrm{~b}$ illustrate the variation of collected hits along the end of the strip implant: the number of collected hits increases around the end of a sensor strip, where the implant is wider, and decreases towards the bias ring, both for an unirradiated and an irradiated sensor. The gap between strip implant and bias ring thus does not provide reliable charge collection.

\section{Conclusion and Outlook}

Measurements of charge collection in the punch-through protection region of a sensor showed little charge collection in the gap between strip implant and bias ring. The punchthrough-protection region thus needs to be treated as dead sensor material.

After measuring the signal collection in the sensor region between the ends of strip implants and bias ring, future measurements are planned to assess the signal collection between the outermost sensor strips and the sensor bias ring (in the region parallel to the strip direction) as well.

\section{Acknowledgements}

The research presented here was supported and financed in part by the UK's Science and Technology Facilities Council; the USA Department of Energy, Grant DE-SC0010107 and DEFG02-13ER41983; the European Union's
Hits in channels $207+208$

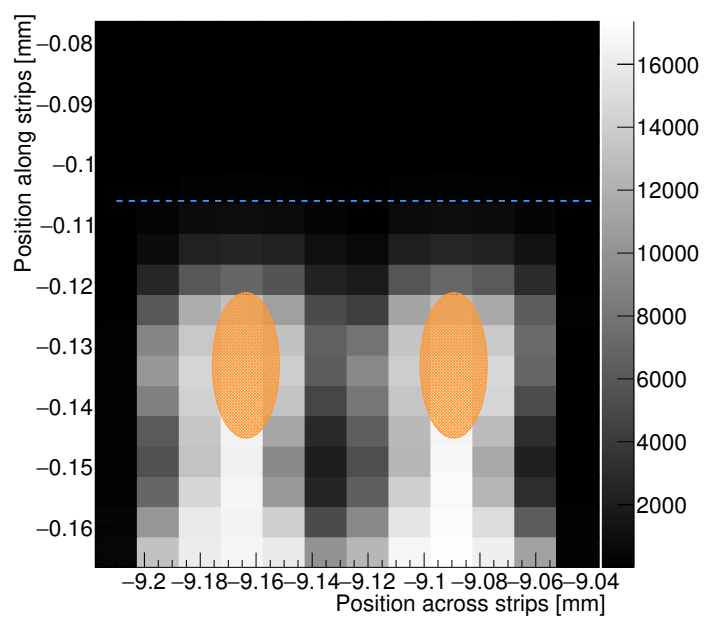

(a) Unirradiated sensor

Hits in channels $97+98$

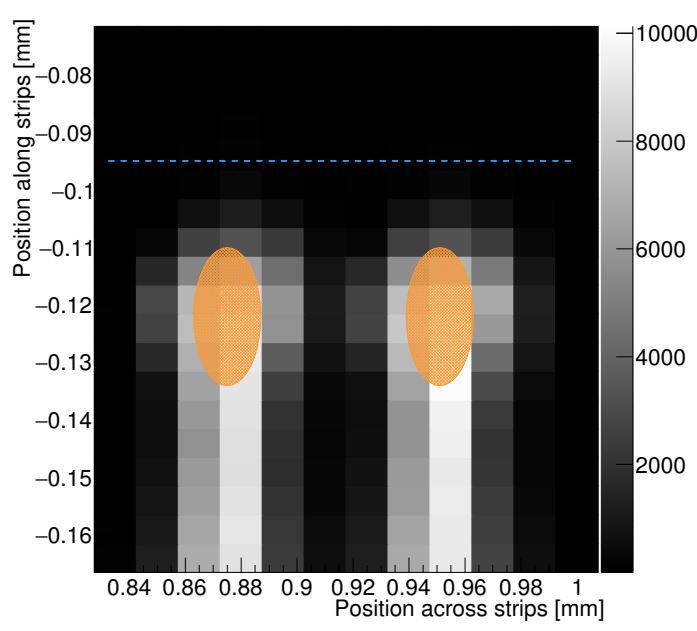

(b) Irradiated sensor

Figure 3: Hit maps from fine scans on an unirradiated sensor (left) and an irradiated sensor (right), showing hits from two adjacent channels each. Between the circular end of the strip implant (orange) and the bias ring (blue), few hits are collected. 


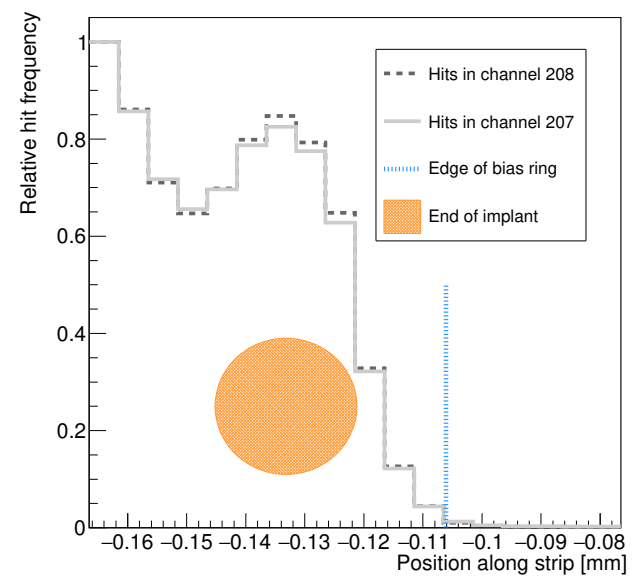

(a) Unirradiated sensor

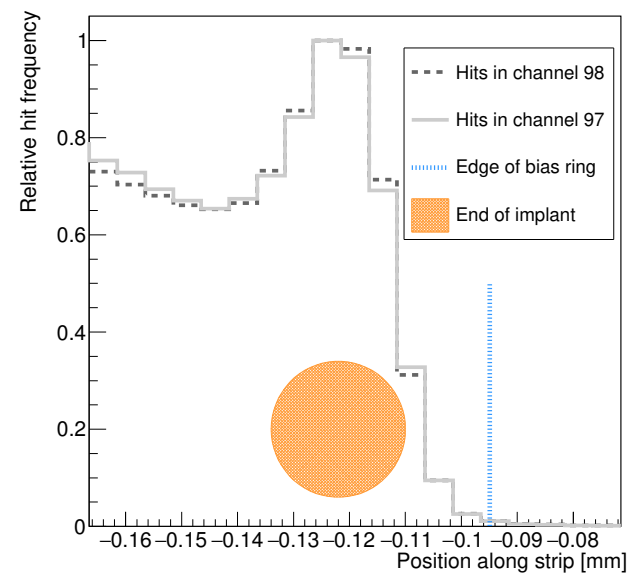

(b) Irradiated sensor
Figure 4: 1-dimensional projection of hits collected 200 along two sensor strips in the region between end of 201 strip implant (orange) and bias ring (blue)
Horizon 2020 Research and Innovation programme under Grant Agreement no. 654168 and Helmholtz CAS Joint Research Grant HCRJG-300. The authors would like to thank Dr. Vladimir Cindro from Jožef Stefan Institute (JSI) for his help with sensor irradiations and the Diamond Light Source for access to beam line B16 (proposal number MT15979) and the personnel of the B16 beam, especially Ian Pape, Oliver Fox and Andy Malandain, for providing advice and support during the experiment.

\section{References}

[1] The ATLAS Collaboration. The ATLAS Experiment at the CERN Large Hadron Collider. Journal of Instrumentation, 3(08):S08003, 2008.

[2] Burkhard Schmidt. The High-Luminosity upgrade of the LHC: Physics and Technology Challenges for the Accelerator and the Experiments. Journal of Physics: Conference Series, 706(2):022002, 2016.

[3] K. Hara, N. Hamasaki, Y. Takahashi, S. Mitsui, Y. Ikegami, Y. Takubo, S. Terada, and Y. Unno. Design of Punch-Through Protection of Silicon Microstrip Detector against Accelerator Beam Splash. Physics Procedia, 37:838 - 843, 2012. Proceedings of the 2nd International Conference on Technology and Instrumentation in Particle Physics (TIPP 2011).

[4] The ATLAS Collaboration. Technical Design Report for the ATLAS Inner Tracker Strip Detector. Technical Report CERN-LHCC-2017-005. ATLASTDR-025, CERN, Geneva, 042017.

[5] K. J. S. Sawhney et al. A Test Beamline on Diamond Light Source. AIP Conference Proceedings, 1234(1):387-390, 2010.

[6] C. Lacasta et al. Design of the first full size ATLAS ITk strip sensor for the endcap region. To be published in Nuclear Instruments and Methods in Physics Research Section A: Accelerators, Spectrometers, Detectors and Associated Equipment, 2018.

[7] R. Marco-Hernandez. A Portable Readout System for Microstrip Silicon Sensors (ALiBaVa). IEEE Transactions on Nuclear Science, 56(3):1642-1649, 2009.

[8] L. Poley et al. Investigations into the impact of locally modified sensor architectures on the detection efficiency of silicon micro-strip sensors. Journal of Instrumentation, 12(07):P07006, 2017. 